\title{
Mobile Assessment of Heightened Skin Conductance in Posttraumatic Stress Disorder
}

Rebecca Roffman ${ }^{1}$, Vasiliki Michopoulos ${ }^{1,2}$, Sterling Winters ${ }^{1}$, Alex 0 Rothbaum ${ }^{3}$, Barbara 0
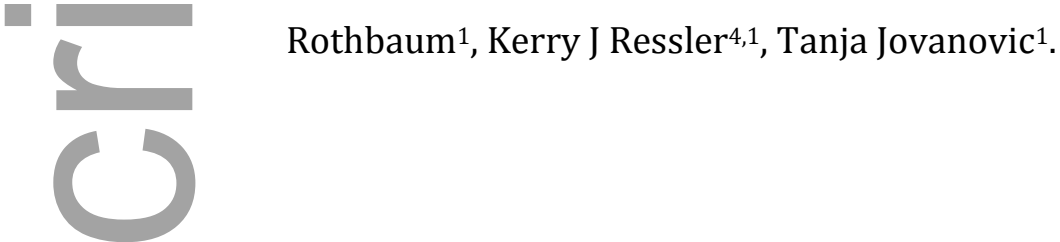

1Department of Psychiatry and Behavioral Sciences, Emory University School of Medicine, Atlanta, Georgia; ${ }^{2}$ Yerkes National Primate Research Center, Atlanta, Georgia; ${ }^{3}$ Case Western Reserve University, Cleveland, Ohio; ${ }^{4}$ Harvard/McLean Hospital, Boston, Massachusetts

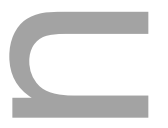

Running Head: Mobile Psychophysiological Assessment of PTSD

Words: 2,820

Tables: 1

Figures: 2

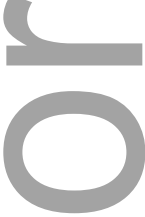

Corresponding Author:

Tanja Jovanovic, $\mathrm{PhD}$

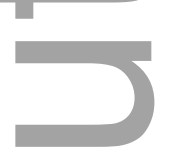

This is the author manuscript accepted for publication and has undergone full peer review but has not been through the copyediting, typesetting, pagination and proofreading process, which may lead to differences between this version and the Version of Record. Please cite this article as doi: 10.1002/da.22610.

This article is protected by copyright. All rights reserved. 
Department of Psychiatry and Behavioral Sciences

Emory University School of Medicine

49 Jesse Hill Jr. NE, Atlanta, GA 30303

E-mail: tjovano@emory.edu

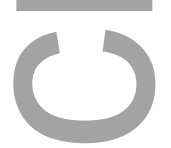

The authors report no conflicts of interest. The work was supported by funding from NIH.

Abstract

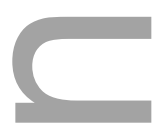

Background: Increased psychophysiological reactivity is a hallmark intermediate phenotype of posttraumatic stress disorder (PTSD). Individuals with PTSD exhibit greater skin conductance (SC) responses to trauma scripts than trauma survivors without PTSD. However, trauma scripts require time for development and cannot be easily used in a single visit. Thus, there is a need for a low-cost, easy-to-use, SC recording protocol for PTSD assessment.

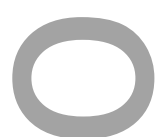

Methods: Using a mobile device (eSense) connected to a portable tablet computer, we assessed SC reactivity to a standard trauma interview (STI) in 63 participants recruited from Grady Memorial Hospital in Atlanta, GA approximately one year after trauma exposure. SC response (SCR) was calculated by subtracting the SCL at the end of the baseline recording from the maximum SC level (SCL) during the STI.

This article is protected by copyright. All rights reserved. 
Results: SCL was significantly higher during the STI compared to baseline $(\mathrm{p}<0.001)$, and individuals with PTSD showed significantly greater SCR than individuals without PTSD $(\mathrm{p}=0.006)$. Logistic regression using SCR with PTSD diagnosis as the outcome showed an odds ratio of 1.76 (95\% CI: 1.11-2.78). Lastly, higher SCR during the STI was also significantly associated with PTSD symptom total score controlling for demographics and trauma severity $(b=0.42, p=0.001)$.

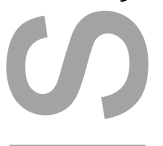

Conclusions: The current study demonstrated feasibility of the use of a mobile device for assessing psychophysiological reactivity in those with PTSD. The use of this low-cost, easy-touse mobile device to collect objective physiological data in concert with a standardized trauma interview can be easily disseminated in clinical and research settings.

\section{Introduction}

Post-traumatic stress disorder (PTSD) is a heterogeneous psychiatric disorder characterized by re-experiencing, avoidance/numbing, and hyper-arousal symptoms [1]. Increased psychophysiological arousal driven by the activation of the autonomic nervous system is a hallmark observation in PTSD [2]. Outputs of autonomic activation, including heart rate (HR), blood pressure (BP), skin conductance (SC), and respiration rate (RR) are all heightened in individuals with PTSD. Therefore using physiological data, such as HR and SC, offers the potential for quantitative objective assessment of physiological reactivity related to PTSD symptoms. One of the earliest methods using trauma-related stimuli to evoke physiological

This article is protected by copyright. All rights reserved. Jovanovic 3 
responses was based on a script-driven imagery procedure developed by Lang and colleagues to study phobias [3]. In this method, the participant describes in detail an experienced traumatic event, which is then transcribed and played back to the individual while physiological responses are recorded [4]. This method has been used in many different combat and civilian PTSD populations [4;5], as well as with women who were sexually abused in childhood [6]. Among the above-mentioned traumatized populations, those with a diagnosis of PTSD exhibit a stronger HR and SC response to scripts than non-PTSD trauma survivors. In 1998, Keane and colleagues [7] conducted the largest script-driven imagery study to date, with almost 1,500 veterans, to look at the utility of psychophysiological measures in diagnosing PTSD. The study concluded that psychophysiological data provided useful and objective assessment of physiological reactivity associated with symptoms of the disorder. However, the specificity for PTSD was not very high, indicating that other factors, such as multiple traumas [8] or high dissociation [9], may be influencing psychophysiological responses. A recent meta-analysis of these early studies confirmed that psychophysiological responses to trauma scripts were predictive of PTSD diagnosis [10] and were stable across time [11]. Finally, both HR and SC show decreased responding after prolonged exposure therapy for PTSD, providing potential objective measures of treatment response [12].

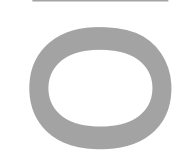

While these data suggest that PTSD can be characterized by psychophysiological reactivity, script-driven imagery methods were developed as experimental protocols and can be burdensome for clinicians, because they entail the development of the trauma scripts over at least two visits and presentation along with neutral scripts. Most studies have used large and elaborate psychophysiological data acquisition equipment that requires dedicated space,

This article is protected by copyright. All rights reserved. 
specialized training, and substantial financial investment. In addition, the methodology has varied across studies, as some studies use multiple trauma scripts, while others use a shortened version. Efforts are currently underway to standardize these methods as common data elements and reduce burden in order to promote the generation of widespread and robust data collection in the same manner (e.g. PhenX Toolkit, www.phenyxtoolkit.org). A novel paradigm that builds on the history of seript-driven imagery but is adapted to be used in clinical settings is recording psychophysiological reactivity during a trauma interview. SC or HR can be recorded continuously during a trauma interview using mobile applications on smartphones and tablets. A recent study that recorded HR while the patient talked about their trauma showed that severity of PTSD symptoms significantly predicted changes in HR, even after controlling for the effects of speech [13]. The importance of this aforementioned study is in the validation of psychophysiological measurements while discussing trauma. In contrast to earlier script-driven imagery, this approach can be easily disseminated in the clinic since a script does not have to be developed and played back to the patient. Such psychophysiological measures can make a seminal contribution to the National Institutes of Mental Health Research Domain Criteria (RDoC) initiative to include biological measures in assessments of mental illness [14; 15]. The measures can both indicate treatment targets and track clinical outcomes in an objective manner [12].

The current study aimed to assess the ability of a low-cost, easy-to-use, SC recording device (eSense) to detect differences in SC response to a standardized trauma interview between trauma-exposed individuals with and without PTSD. In addition, we hypothesized that concurrent PTSD symptom severity would be associated with SC reactivity. The validation of a

This article is protected by copyright. All rights reserved. Jovanovic 5 
cost- and time-effective method to capture physiological indices that can be easily obtained in most clinical and research settings may prove beneficial in the diagnosis and treatment of PTSD.

\section{Methods}

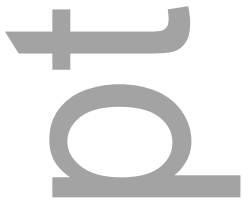

Subjects. Study participants $(\mathrm{n}=63)$ were recruited from the trauma center of Grady Memorial Hospital in Atlanta, GA after having experienced a criterion-A trauma for PTSD [16]. Participants were English-speaking men and women between the ages of 18 and 65 years who provided written informed consent. Exclusion criteria included significant substance use during screen identified by a positive toxicology report in the electronic medical record, current or recent suicidality, active psychosis, or not alert, oriented, and coherent as measured by Glasgow Coma Scale $($ GCS $)<15$. Patients were also excluded for respiratory distress or if medically unstable or hemodynamically compromised. After an initial assessment in the trauma center, participants completed a follow-up assessment approximately one year from the time of trauma exposure. All study procedures were reviewed and approved by the Emory Institutional Review Board and the Grady Hospital Research Oversight Committee. All participants participated in a standardized assessment wherein a standardized trauma interview was conducted by trained research staff.

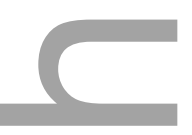

Measures. The PTSD Diagnostic Scale (PDS) and the Standardized Trauma Interview (STI) were used during baseline assessment in the trauma center to determine lifetime trauma history [17; 18]. The STI was repeated one year later in a follow-up session, and the current paper only presents SC data collected during the follow-up visit. The STI contains 21 questions that query

This article is protected by copyright. All rights reserved. 
details about the trauma (for example: "What time of day did it happen?", "How long did this event last?", “Did you see other people's injury or death?”). The PhenX Toolkit lists all questions used in the STI at

https://www.phenxtoolkit.org/index.php?pageLink=browse.protocoldetails\&id=630901.

Current PTSD diagnosis and symptoms were measured by trained interviewers at the 1-year follow-up assessment using the previously validated PTSD Symptom Scale (PSS) [19]. The PSS is a psychometrically valid 17-item self-report scale assessing PTSD symptoms over the past two weeks [20;21]. A PTSD diagnosis (scored as 0 or 1) was based on DSM-IV criteria, if participants met at least one re-experiencing symptom, three avoidance and/or numbing symptoms, two hyperarousal symptoms, and if the duration of symptoms was greater than one month [22]. The interview version of the PSS has been validated with clinician administered instruments and has been used previously to assess PTSD diagnosis [23]. For a continuous measure of PTSD symptom total, we summed the PSS items as previously described [24]. Similarly, we computed continuous measures for sub-clusters of symptoms, including reexperiencing, avoidance and hyperarousal symptom clusters. Inter-rater reliability was greater than $97 \%$ for all administered measures. All data were captured and managed using REDCap electronic data capture tools hosted by Emory University [25].

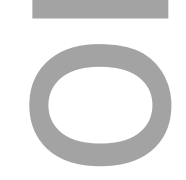

Mobile SC assessment. Skin conductance was assessed using the eSense SC system (Mindfield Biosystems, Inc., Berlin, Germany) on an iPad (iOS10). The eSense software was downloaded to the iPad and two finger electrodes were attached to the middle and index finger with Velcro straps. The electrodes were connected to the iPad using the audio connection input. In order to ensure good contact with the skin, isotonic paste was added to the electrodes prior to attaching

This article is protected by copyright. All rights reserved. 
to the fingers. eSense acquired data at a sampling rate of $10 \mathrm{~Hz}$ and the data were exported via email using csv files. Prior to using eSense in the current clinical assessment for PTSD, the method was tested as a proof-of-concept by comparing SC level (SCL) data collected by eSense to SCL collected concurrently in the same individual using a Biopac system (Biopac Systems, MP150 for Windows, Goleta, CA), with data sampled at $1000 \mathrm{~Hz}$. Briefly, data were collected from a healthy volunteer (independent from current study sample) during a stressful arithmetic task with two isotonic gel-filled electrodes on one hand connected to eSense and electrodes on the other hand connected to Biopac. The electrodes and gel used in the test case were identical to the ones used in the clinical assessment study. The data were highly correlated, $r=0.94$, $\mathrm{p}<0.001$. In the clinical assessment part of the current study, electrodes were attached to the fingers of the participant's non-dominant hand during administration of the STI (Figure 1). Baseline SCL was recorded at the beginning of our standardized assessment during a 2-minute rest period, immediately followed by the STI. SC response (SCR) was calculated by subtracting the SCL at the end of the baseline recording (average of the last 30 seconds of the 2-minute period) from the maximum SCL value during the STI.

Statistics. The data were analyzed using SPSS (v.24) and were summarized as mean \pm standard error of the mean (SEM). The alpha level was set at $\mathrm{p} \leq 0.05$ for statistical significance. The effects of PTSD diagnosis on SCL during the STI were analyzed using separate $2 \mathrm{X} 2$ analysis of variance (ANOVA) with PTSD diagnosis as the between-subjects factor, and condition (baseline vs. STI) as the within-subjects factor. Pearson's correlations were used to summarize the relationship between SCR and PTSD symptom severity, as well as with PTSD symptom subclusters. Linear regression was used to determine whether peak SCR to the STI was predictive

This article is protected by copyright. All rights reserved. 
of continuous PTSD symptom severity, and logistic regression was used to determine whether peak SCR predicted categorical PTSD diagnosis. Finally, Receiver Operating Characteristic Curve (ROC) was used to evaluate the clinical performance for detection of PTSD by SCR. The areas under the curve and 95\% confidence intervals were determined to test the accuracy of SCR for predicting PTSD diagnosis.

\section{Results}

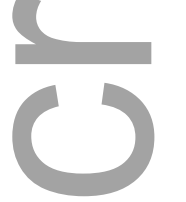

Sociodemographics. The mean \pm SEM age in the overall sample was $34.8 \pm 1.82$ and participants had experienced $2.58 \pm 0.23$ traumas in their lifetime. Traumas experienced and queried in relationship to current PTSD symptoms included: non-sexual assaults (9.5\%), motor vehicle collisions (50.8\%), sexual assaults (7.9\%), motorcycle accidents (14.3\%), and other $(17.5 \%)$. The mean \pm SEM time since index trauma exposure in the overall sample was $386 \pm 3.14$ days. The participants were primarily African American, with an average monthly income of $>\$ 1000$. Men and women were equally represented in the current study. Of the 63 participants, 15 met diagnostic criteria for PTSD. Table 1 shows the demographic and trauma history information across participants with and without PTSD. There were no significant differences in these factors between PTSD+ and PTSD- groups (all p's>0.05).

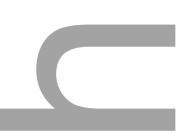

PTSD Diagnosis and SCR to trauma reminder. SCL was significantly higher during the STI compared to baseline $\left(\mathrm{F}_{1,61}=67.8, \mathrm{p}<0.001\right)$. While there was no main effect of PTSD diagnosis on average SCL $\left(\mathrm{F}_{1,61}=3.75, \mathrm{p}=0.057\right)$, there was a significant condition by PTSD interaction $\left(F_{1,61}=8.25, p=0.006\right)$. Individuals with PTSD showed significantly higher peaks in SCL during

This article is protected by copyright. All rights reserved. Jovanovic 9 
the STI compared to individuals without PTSD (Figure 2A). In addition, the SCR to the STI was significantly greater in those with PTSD compared to those without PTSD $\left(\mathrm{F}_{1,61}=8.25, \mathrm{p}=0.006\right.$; Figure 2B). This effect of PTSD remained significant after controlling for demographic variables, including age, sex, and trauma exposure $\left(\mathrm{F}_{1,58}=7.06, \mathrm{p}=0.01\right)$. Logistic regression using the SCR with PTSD diagnosis as the outcome showed an odds ratio of 1.76 (95\% CI, 1.11-2.78), indicating that for each $1 \mu \mathrm{S}$ increase in SC change score in response to trauma challenge, there was a 76\% greater odds of having a PTSD diagnosis. The AUC for the ROC curve analysis for SCR on PTSD diagnosis was 0.79 (p=0.001), with 95\% confidence intervals of 0.66 and 0.91 .

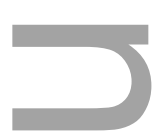

PTSD symptom total and SCR to trauma reminder. PTSD symptom total was significantly correlated with SCR to the STI ( $\mathrm{r}=0.42, \mathrm{p}=0.001)$, Figure 2C. SCR during the STI was also significantly associated with symptom cluster subscales: intrusive $(r=0.36, p=0.004)$, avoidance/numbing ( $r=0.38, \mathrm{p}=0.002)$, and hyperarousal symptoms ( $r=0.38, \mathrm{p}=0.002)$. Linear regression showed that the increase in SCR was significantly predictive of PTSD symptom total after controlling for covariates, including age, sex, and trauma exposure $(b=0.42, p=0.001)$.

\section{Discussion}

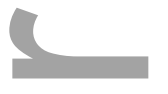

The current study found that changes in skin conductance elicited by a standardized trauma interview were greater in individuals with PTSD compared to those without PTSD. There was also a significant positive correlation between SCR and PTSD symptoms. While SC has been shown in previous studies to be associated with PTSD symptoms [4-6; 10], the current study is an important extension of previous work in three ways: 1) it used a low-cost mobile device to

This article is protected by copyright. All rights reserved. 
collect SC data, 2) the method can be easily disseminated in a wide variety of clinical and research settings, and 3) it used a standardized trauma interview in combination with psychophysiological assessment, which was developed as a common data element for PTSD in order to standardize PTSD measures across different settings.

$\square$

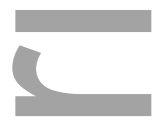

Our data show that psychophysiological responses can be measured using low-cost and lowburden instruments, which are critical for the widespread dissemination of these objective measures to assess psychophysiological reactivity in those with PTSD. Importantly, we first tested this mobile method as a proof-of-concept by comparing individual results with state-ofthe-art research equipment, Biopac Systems MP150 that we have used in our previous research [26; 27]. In addition, we validated the use of a standardized psychophysiological trauma challenge measure from the PhenX Toolkit, which is available for download. While the STI has been validated clinically [18], this is the first time it has been used as a psychophysiological challenge task. Because such psychophysiological tools provide objective, concomitant measures for the assessment of PTSD symptoms, they should be useful in clinical applications, preclinical research, and clinical trials to measure quantitative biological outcomes in addition to symptom ratings. SCR has shown promise in assessment of PTSD symptoms in clinical trials [26], but to date recording SCR has been burdensome for clinical use. Taking advantage of novel technology may encourage the field to incorporate more of these methods into practice. Given that the odds ratio indicated that an increase of one $\mu \mathrm{S}$ during the trauma reminder increased

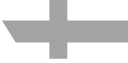
odds of PTSD diagnosis by $76 \%$ and the ROC AUC was 0.79 , this biological assessment method could provide a useful tool. With increasing emphasis on the use of biological measures in

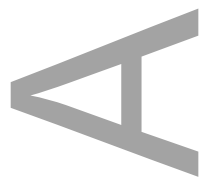

This article is protected by copyright. All rights reserved. 
mental health consistent with RDoC, future research and clinical settings can greatly benefit from validated and clinically relevant psychophysiological tools.

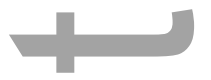

To our knowledge, the current study is the first published account of using this mobile technology to assess psychophysiological reactivity in PTSD, thereby providing important proof-of-concept. While previous data show that SC measurement may be more difficult to assess in African American individuals [28; 29], race did not appear to affect either SCL or SCR in the current study ( $\mathrm{p}=0.22$ and $\mathrm{p}=0.24$, respectively). Replication studies in larger sample sizes must be undertaken to assess the utility and generalizability of this approach alongside other measures of autonomic function, and allow for further characterization of factors that might influence PTSD symptoms, as SCR alone had a specificity of 79\% for PTSD. Thus, a few individuals with low PTSD symptoms demonstrated high SCR to trauma reminders (Figure 2C). These replications would also address one of the limitations of the current study; specifically, that it includes a relatively small proportion of PTSD+ cases. However, by also assessing the relationship between SCR and continuous PTSD symptoms, we have included a wider range of pathology and a more dimensional approach in our analyses. A further limitation of the current study was that it was based on DSM-IV criteria for PTSD [16], given that it was started prior to the change to DSM-5 [30]. Another limitation that should be addressed in future validation studies is that SC was collected during the interview and could have been impacted by speech. While a previous study of HR did not show detrimental impact of speech patterns, SC may be more easily affected by changes in respiration. A validation study using an imagery component after the interview might minimize this effect. Further, it is possible that those with higher symptoms would have more disrupted speech patterns. Future studies might separate these

This article is protected by copyright. All rights reserved. 
mechanistic effects; in the interim, the utility of the measure in clinical settings appears very promising.

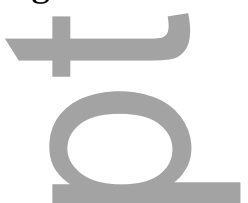

In summary, the current study presents a simple and novel method of assessing physiological reactivity to trauma-related cues using a mobile device to capture SC responses without the need of specialized training. While this method is not intended to replace clinician or patient symptom ratings, it can be used in conjunction with such ratings to provide a biological measure of symptom severity that is easily measured and readily accessible.

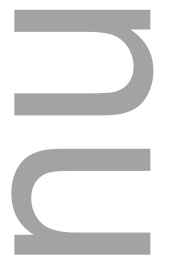

\section{Acknowledgments}

We would like to acknowledge the participants that agreed to be a part of this study. This work was supported by the Research and Woodruff Health Sciences IT Division (UL1 TR000424), Howard Hughes Medical Institute (KJR), R01 MH094757 (KJR), K12 HD085850 (VM), R21 MH106902 (TJ), and the Brain and Behavior Research Foundation. The funding sources had no role in the design and conduct of the study; collection, analysis, and interpretation of the data; preparation, review, or approval of the manuscript; and decision to submit the manuscript for publication. A special thanks to Peter Tuerk, Ph.D. for introducing us to the eSense software and giving us our first set of electrodes.

\section{References}

1.Kessler RC, Sonnega A, Bromet E et al. . Posttraumatic stress disorder in the National Comorbidity Survey. Arch Gen Psychiatry 1995;52(12):1048-60.

This article is protected by copyright. All rights reserved. Jovanovic 13 
2.Michopoulos V, Norrholm SD, Jovanovic T. Diagnostic Biomarkers for Posttraumatic Stress Disorder: Promising Horizons from Translational Neuroscience Research. Biol Psychiatry 2015;78(5):344-53.

3.Lang PJ. Anxiety: Toward a psychophysiological definition. In: Akiskal HS, Webb WL, editors.

Psychiatric Diagnosis: Exploration of Biological Predictors. New York: Spectrum; 1978, p 365-389.

4.Pitman RK, Orr SP, Forgue DF et al. . Psychophysiologic assessment of posttraumatic stress disorderimagery in Vietnam combat veterans. Arch Gen Psychiatry 1987;44(11):970-5.

5.Shalev AY, Orr SP, Pitman RK. Psychophysiologic assessment of traumatic imagery in Israeli civilian patients with posttraumatic stress disorder. Am J Psychiatry 1993;150(4):620-4.

6.Orr SP, Lasko NB, Metzger LJ et al. . Psychophysiologic assessment of PTSD in adult females sexually abused during childhood. Ann N Y Acad Sci 1997;821:491-3.

7.Keane TM, Kolb LC, Kaloupek DG et al. . Utility of psychophysiological measurement in the diagnosis of posttraumatic stress disorder: results from a Department of Veterans Affairs Cooperative Study. J Consult Clin Psychol 1998;66(6):914-23.

8.McTeague LM, Lang PJ, Laplante MC et al. . Aversive imagery in posttraumatic stress disorder: trauma recurrence, comorbidity, and physiological reactivity. Biol Psychiatry 2010;67(4):346-56.

9.Griffin MG, Resick PA, Mechanic MB. Objective assessment of peritraumatic dissociation: psychophysiological indicators. Am J Psychiatry 1997;154(8):1081-1088.

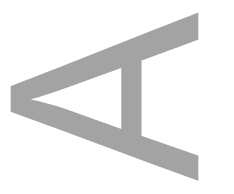

This article is protected by copyright. All rights reserved. Jovanovic 14 
10.Pineles SL, Suvak MK, Liverant GI et al. . Psychophysiologic reactivity, subjective distress, and their associations with PTSD diagnosis. J Abnorm Psychol 2013;122(3):635-44.

11.Bauer MR, Ruef AM, Pineles SL et al. . Psychophysiological assessment of PTSD: a potential research domain criteria construct. Psychol Assess 2013;25(3):1037-43.

12.Wangelin BC, Tuerk PW. Taking the Pulse of Prolonged Exposure Therapy: Physiological Reactivity to Trauma Imagery as an Objective Measure of Treatment Response. Depress Anxiety 2015;32(12):927-34.

13.Nachar N, Guay S, Beaulieu-Prevost D, Marchand A. Assessment of the Psychosocial Predictors of Health-Related Quality of Life in a PTSD Clinical Sample. Traumatology (Tallahass Fla) 2013;19(1):20-27.

14.Miller GA, Rockstroh BS, Hamilton HK, Yee CM. Psychophysiology as a core strategy in RDoC. Psychophysiology 2016;53(3):410-4.

15.Morris SE, Vaidyanathan U, Cuthbert BN. Psychophysiological science and the research domain criteria: A commentary. Int J Psychophysiol 2015;98(2 Pt 2):378-80.

16.APA. Diagnostic and Statistical Manual of Mental Disorders. 2000.

17.Foa EB, Rothbaum B. Treating the Trauma of Rape: Cognitive-behavioral therapy for PTSD. New York: The Guilford Press. 1998.

18.Rothbaum B, Foa EB, Riggs DS et al. . A Prospective Examination of Post- traumatic Stress Disorder in Rape Victims. Journal of Traumatic Stress 1992;(5):455-475.

19.Foa EB, Riggs DS, Dancu CV, Rothbaum BO. Reliability and validity of a brief instrument for assessing post-traumatic stress disorder. Journal of Traumatic Stress 1993;(6):459-473.

This article is protected by copyright. All rights reserved. Jovanovic 15 
20.Schwartz AC, Bradley RL, Sexton M et al. . Posttraumatic stress disorder among African Americans in an inner city mental health clinic. Psychiatr Serv 2005;56(2):212-5.

21.Foa EB, Tolin DF. Comparison of the PTSD symptom scale-interview version and the clinician-administered PTSD scale. Journal of Traumatic Stress 2000;13(2):181-191.

22.Falsetti SA, Resnick HS, Resick PA, Kilpatrick DG. The Modified PTSD Symptom Scale: A brief self-report measure of posttraumatic stress disorder. . Behaviour Therapist 1993;(16):161-161.

23.Jovanovic T, Norrholm SD, Blanding NQ et al. . Impaired fear inhibition is a biomarker of PTSD but not depression. Depression and Anxiety 2010;27(3):244-251.

24.Gillespie CF, Bradley B, Mercer K et al. . Trauma exposure and stress-related disorders in inner city primary care patients. Gen Hosp Psychiatry 2009;31(6):505-14.

25.Harris PA, Taylor R, Thielke R et al. . Research electronic data capture (REDCap)--a metadatadriven methodology and workflow process for providing translational research informatics support. J Biomed Inform 2009;42(2):377-81.

26.Rothbaum BO, Kearns MC, Reiser E et al. . Early intervention following trauma may mitigate genetic risk for PTSD in civilians: a pilot prospective emergency department study. J Clin Psychiatry 2014;75(12):1380-7.

27.Glover EM, Phifer JE, Crain DF et al. . Tools for translational neuroscience: PTSD is associated with heightened fear responses using acoustic startle but not skin conductance measures. Depress Anxiety 2011;28(12):1058-66.

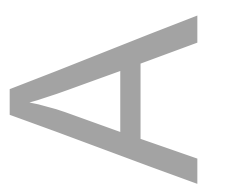

This article is protected by copyright. All rights reserved. Jovanovic 16 
28.Johnson LC, Landon MM. Eccrine sweat gland activity and racial differences in resting skin conductance. Psychophysiology 1965;1(4):322-9.

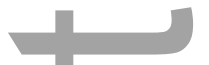

29.Lieblich I, Kugelmass S, Ben-Shakhar G. Psychophysiological baselines as a function of race and ethnic origin. Psychophysiology 1973;10(4):426-30.

30.APA. Diagnostic and Statistical Manual of Mental Disorders. 2014.

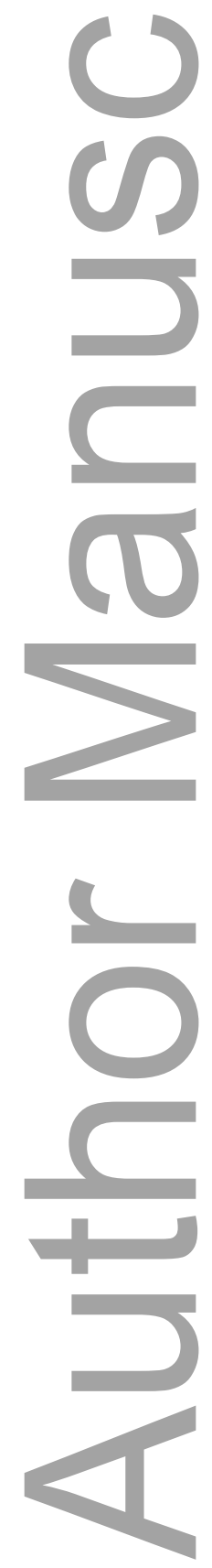

This article is protected by copyright. All rights reserved. 
Table 1. Mean \pm SEM and frequency (\%) of participant sociodemographic characteristics.

\begin{tabular}{|c|c|c|c|}
\hline & PTSD + (n=15) & PTSD - $(n=48)$ & p-value \\
\hline Age (years) & $33.2 \pm 3.00$ & $35.3 \pm 2.21$ & 0.62 \\
\hline $\begin{array}{l}\text { Time from Index } \\
\text { Trauma (days) }\end{array}$ & $393.9 \pm 8.02$ & $383.5 \pm 3.25$ & 0.16 \\
\hline $\begin{array}{l}\text { Number of Lifetime } \\
\text { Traumas }\end{array}$ & $3.06 \pm 0.52$ & $2.44 \pm 0.25$ & 0.25 \\
\hline Race & & & 0.29 \\
\hline African American & 12 & 36 & \\
\hline White/Caucasian & 1 & 8 & \\
\hline Other & 2 & 2 & \\
\hline Sex & & & 0.82 \\
\hline Male & 8 & 24 & \\
\hline Female & 7 & 24 & \\
\hline Monthly Income & & & 0.73 \\
\hline$<\$ 500$ & 1 & 8 & \\
\hline$\$ 500-\$ 999$ & 4 & 9 & \\
\hline$>\$ 1000$ & 9 & 29 & \\
\hline
\end{tabular}

Figure 1. Mobile SC assessment using eSense device and iPad

This article is protected by copyright. All rights reserved. 


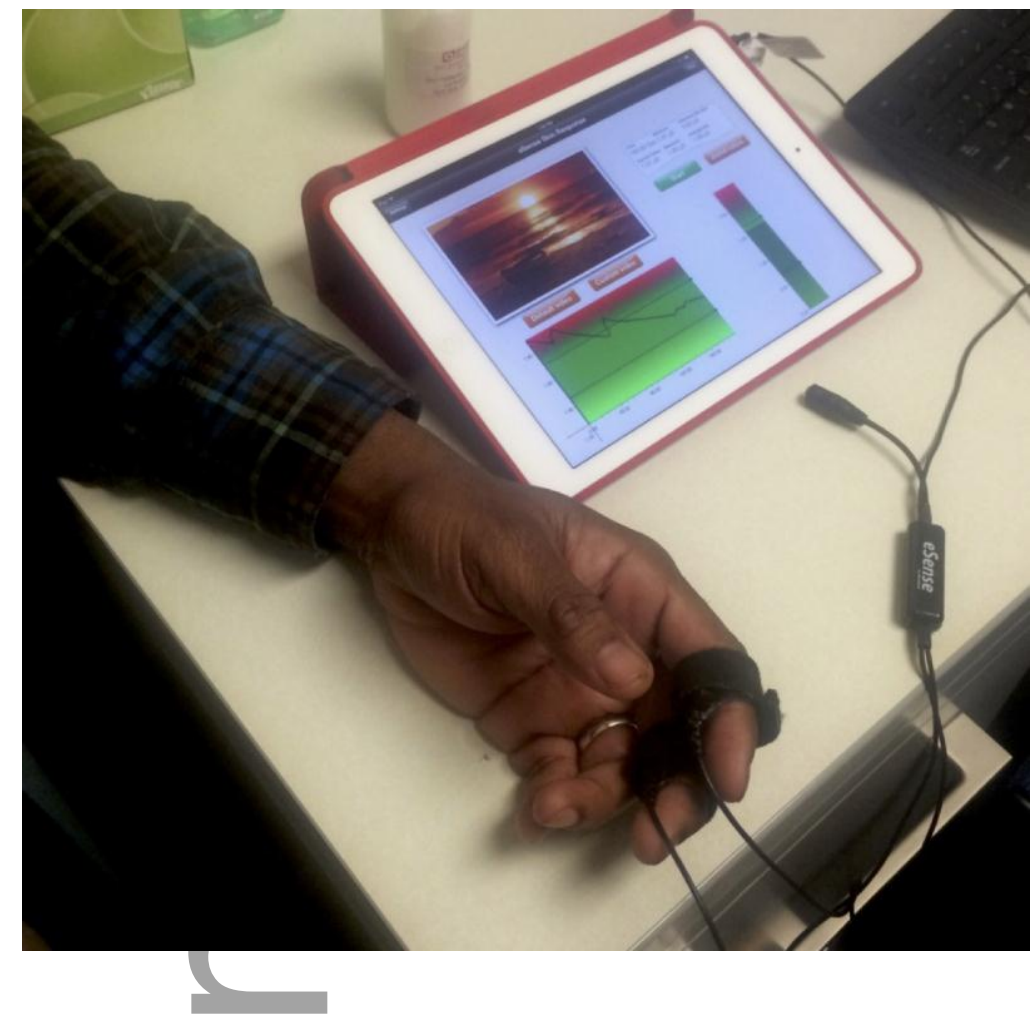

Figure 2. (A) Mean \pm SEM skin conductance level (SCL, microSiemems, $\mu$ S) at baseline and at maximum levels during trauma reminder broken down by PTSD diagnosis. . (B) The increase in SCR to STI in PTSD+ and PTSD- individuals. (C) Scatter plot of PTSD symptoms (PSS total score) and SCR to STI.

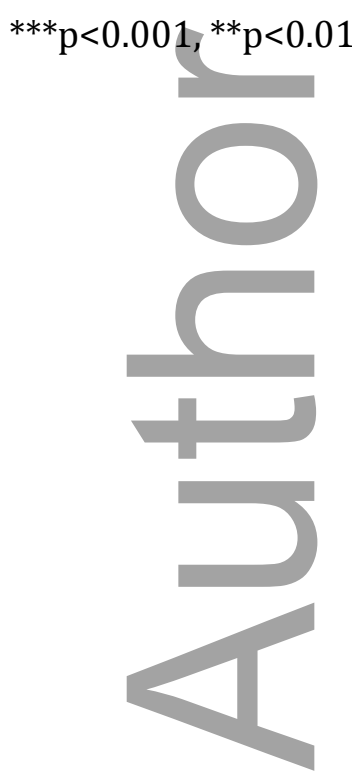

This article is protected by copyright. All rights reserved. 


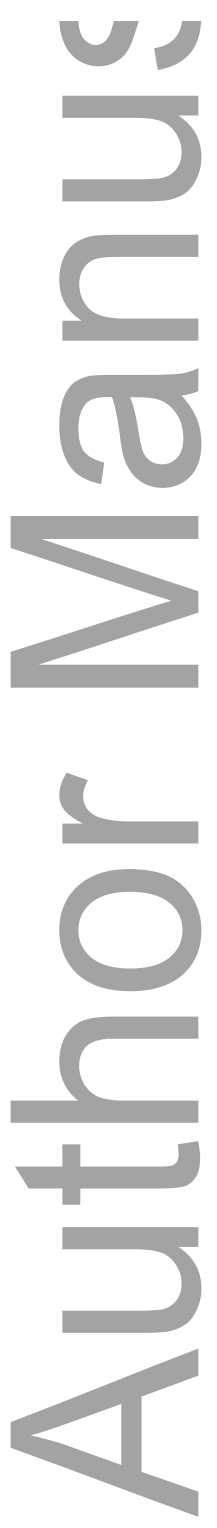

This article is protected by copyright. All rights reserved.

Jovanovic 20 